\title{
A Survey on Intermittent Fault Diagnosis for Electronic System
}

\author{
Husheng $\mathrm{Wu}^{1,}$, , Sheng Sheng', b, Qian Yan ${ }^{1, \mathrm{c}}$, Dongli Duan ${ }^{1, \mathrm{c}}$ \\ ${ }^{1}$ Materiel Engineering College, Armed Police Force Engineering University, Xi'an 710086, China \\ ${ }^{2}$ Materiel Management and Safety Engineering College, Air Force Engineering University, Xi'an \\ 710051, China \\ awuhusheng0421@163.com, bafeusheng@126.com, 'mineduan@163.com
}

Keywords: Electronic system, intermittent fault, Fault diagnosis, Fault detection

\begin{abstract}
The intermittent fault of electronic system is often misdiagnosed as a permanent fault, resulting in problems such as unnecessary repeated downtime, excessive repair and a large loss of spare parts, and affecting equipment readiness. Starting from the overall development of intermittent fault for electronic system to tease out the research direction, this paper focuses on the research of intermittent fault for electronic system from four aspects: the failure mode, cause and mechanism analysis, modeling method, detection method and diagnosis / prediction method, and points out the shortcomings of the current research on intermittent fault of electronic system.
\end{abstract}

\section{Introduction}

Electronic products have penetrated into all walks of life. According to the prediction of International Technology Roadmap for Semiconductors (ITRS), the complexity and technological level of electronic products also shows a strong upward trend, followed by a variety of fault threats. The faults of electronic systems, according to the time characteristics, can be classified into three types [1]: permanent fault, transient fault and intermittent fault. 1) Permanent fault refers to the fault which can't return to be normal by itself from fault state, and often influences the functional behavior of the system continuously and steady. 2) Transient fault is caused by the particle radiation, electromagnetic wave and power-supply fluctuation, and has no fault of any component. It often occurs instantaneously. 3) Intermittent fault can return to be normal from fault state by itself, and it usually occurs at the same position repeatedly once occurred, showing the intermittent characteristics that occurs from time to time. Transient faults are mostly related to the external disturbances and have real randomness. And permanent faults have formed a complete set of theoretical system. Both of them will not be discussed in the paper.

As a special fault form, the intermittent fault is random, intermittent, repetitive and cumulative, seriously affecting the performance of electronic equipment. For example, in the mixed circuit, intermittent fault is the main cause of temporary failure. Even in the ideal environment, intermittent faults would be 10 to 30 times the number of permanent faults. Digital circuit system dysfunction is mainly caused by intermittent faults, accounting for $90 \%$ of all the possible faults. But abnormal performance usually disappears after the system reboot, bring difficulties to fault detection. In the large scale integrated circuit, a permanent fault occurs on average every 7700 hours, while an intermittent fault occurs on average every 100 hours [2]. Especially with the constant injection of high-tech and the increasing complexity of electronic system, the intermittent fault has become even more prominent, especially for the electronic devices in harsh environments, such as island, desert and plateau. Related research shows that intermittent fault is one of the important factors which cause the low detection rate and high false alarm rate. It directly leads to unnecessary repairs, low operational readiness and premature system replacement, resulting in a huge waste of resources. However, due to the particularity of intermittent faults, it is difficult to apply the existing fault diagnosis methods to intermittent faults which are difficult to be suppressed and blanked efficiently. In addition, some experts, organizations or institutions also point out the inefficiency of the existing methods in response to intermittent faults. Pecht points out that nonpermanent faults such as intermittent faults are the "weakness" in the field of Prognostics and Health Management (PHM). It 
can be seen that the intermittent fault of electronic system and its fault mode, mechanism, detection and diagnosis method are very worthy of further researching. Based on this, the author studies the intermittent fault of electronic system.

\section{Overall Trend Analysis of The Study on Intermittent Fault For Electronic System}

Early in the 1940s and 1950s, people have carried out to study problems such as arc of fire which is caused by intermittent short circuit between the cable and the casing on machine, including not only the analysis of the principle, but also the study of experimental methods and detection methods. Now, the research on intermittent faults has accumulated certain achievement. Through the search and analysis of some databases, it is not difficult to see the overall development trend. Taking "IEEE Xplore Digital Library" and "Science Direct" as an example, "intermittent failure" and "intermittent fault" are used as keywords (the relationship of the two keywords is "OR"), the search condition is set to " (Document) Title, Abstract, Index Terms/Key Words ", and retrieve the unrelated literature by browsing" Abstract ". Column charts shown in Figure 1can be achieved.
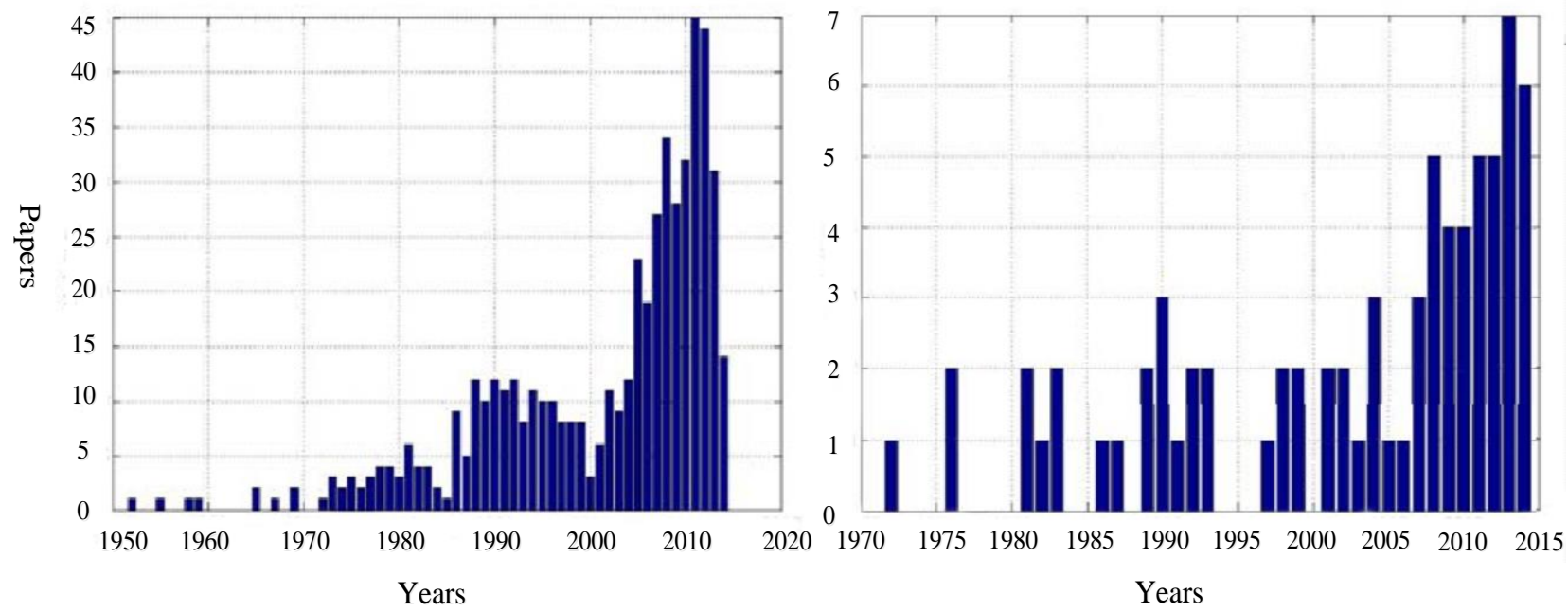

Fig. 1 The distribution of the number of documents related to intermittent faults in the two databases

(Data on the left are from IEEE Xplore Digital Library, and data on the right are from Science Direct)

It can be seen from Figure 1 that the intermittent fault research in the 1950s and 1960s is relatively sporadic, and it is not very concerned. After the 1970s, the study of the intermittent fault has an overall tendency to be popular. Around 2008, the attention on the intermittent fault has been greatly improved. Since the statistics given in Figure 1 can't cover all the literature related to intermittent faults, it is impossible to take the absolute number of documents in the figures for analysis. But obviously, compared with the number of other literature in the field of PHM, there are few literature on the intermittent fault. It can be seen from several held International Conferences on the theme of PHM: From 2008 to 2014, IEEE has organized nine international conferences themed "Prognostics and Health Management" and "Prognostics and System Health Management". Although only 5 in all of the 765 papers are related to the intermittent fault, the higher destructive force which has been shown and the current attention which is not timely follow-up are doomed that the problem of the intermittent fault will be more and more popular as shown in Figure 1.

\section{Main Research Direction of Intermittent Fault For Electronic System}

The research directions of the literature can be collected and classified by consulting relevant literature in the database and part of the references, as shown in table 1.

Table 1 shows that fault modes, causes, and mechanism analysis and detection methods are mostly researched. For some intermittent faults of which the cause and mechanism are complex and fault phenomenon can't be observed obviously, dozens of literature are retrieved merely for this kind of 
intermittent faults. The reason why the two parts account for a large proportion should be the most urgent need for these two aspects: On one hand, people need to study the fault modes, causes and mechanism to deeply understand the intermittent fault. On the other hand, effective detection methods are needed urgently to detect and isolate intermittent faults. Here is the literature review for abovementioned four main respects.

Table 1. Main research direction and proportional distribution of literatures related to intermittent faults

$\begin{array}{ll}\text { Main research direction } & \text { Proportion } \\ \text { Fault mode, cause and mechanism analysis } & 65.1 \% \\ \text { Modeling method } & 6.2 \% \\ \text { Detection method } & 26.1 \% \\ \text { Diagnosis/prediction method } & 7.2 \% \\ \text { Others } & 3.6 \%\end{array}$

\subsection{Fault Mode, Cause And Mechanism Analysis}

Intermittent fault mode, cause and mechanism analysis are mostly realized by some basic experiments. The results of the research are around the laboratories, research institutes, universities, companies, military and state-owned units all over the world, such as research on intermittent faults caused by the conductive wire by the University of Maryland, Honeywell and Rockwell [3], research on intermittent faults caused by the contact resistance by Chrysler and the University of Lyon in France [4], research on intermittent faults caused by creep fatigue by Atomic Energy Research Institute in Korea and the University of Strathclyde in British [5], research on intermittent faults caused by the electrochemical migration by the Technology University of Denmark and the University of Science and Technology Beijing [6], research on intermittent faults caused by the friction corrosion by Korea POSCO Technology Laboratory, the Seoul's Yonsei University, the Harbin Institute of Technology and the Germany East Westphalia - Lipp University of Applied Technology [7], research on intermittent faults caused by the electronic "lead-free" by the University of Maryland, the Institute of Electronic Technology in Poland, the Korea Yeo University, Samsung, the Michigan State University, the University of Science and Technology Beijing and the University of Hong Kong [8], research on intermittent faults caused by the solder joint by the National Tsinghua University in Taiwan, Thales in French, the Bordeaux First University in France and the Air Force Engineering University [9], research on intermittent faults caused by the tin/metal whisker by NASA, the University of Maryland, the Korea Yeo University, Korea Electronics and Telecommunications Research Institute, Korea National Nanotechnology Manufacturing Center, Samsung, Los Angeles Air Force Base, Lucent Technology in America, Bell Labs, Raytheon, National Taiwan University of Science and Technology, National Taiwan University and Institute of Metal Research, Chinese Academy of Sciences [10], research on intermittent faults caused by the wafer bonding of gold by NASA, the Technical University of Munich in Germany, Switzerland ABB company and Chongqing University and research on intermittent faults caused by the cable connection by the advanced automatic equipment company [11].

However, the cause and mechanism of some intermittent faults can't be fully understood. For example, there is no single and widely accepted explanation of the generation/growth mechanism of tin/metal whisker causing intermittent faults. 7 International Symposiums on Tin Whiskers have been held to promote the study of tin whisker and strengthen the exchange of research results.

\subsection{Modeling Method}

The modeling method for the intermittent fault of electronic systems is mainly carried out on a series of characteristics of intermittent faults. For example, the on-off characteristics of the intermittent open circuit fault are simulated by the equivalent circuit model ("switch + variable resistor") in [12]. Another equivalent circuit model (variable resistor) is used to simulate the on-off characteristics of the intermittent open circuit fault. Alpha count model is used to determine a related fault type "alpha index" to distinguish the intermittent fault from other types of faults, such as the permanent fault and transient fault in [13]. Markov model is used to describe the transfer of the intermittent fault from the normal state to the fault state in [14]. The finite state machine is used to 
describe the transfer of the intermittent fault from the normal state to the fault state in [15]. In [16], the intermittent fault is written into the observation equation to analyze it by the state equation model. The Bayesian model based on probability is used to describe the probability of the intermittent fault part in the normal state in [17].

\subsection{Detection Method}

Traditional detection methods are often inadequate when dealing with intermittent faults. At present, a number of detection methods have been developed specifically for intermittent faults of electronic systems and have been successfully applied, but only focused on intermittent faults caused by partial causes/mechanisms. For example, various reflectometers have been widely used in the cable path detection, and have a very good effect on the intermittent connection fault. Characteristics and principles of various reflectometers and an inspection tester- wire integrity tester (WIT) based on various reflectometers are introduced in [18]. The application of the reflectometer in intermittent fault detection of the flight control system for F-18 fighter is introduced in [19]. The application of the reflectometer in the field of civil aviation and military is introduced in [20] and the application of time and frequency domain reflectometer in the business is introduced in [21].Given the difficulty traditional ATE dealing with, Universal Synaptic company in America designed intermittent fault detector (IFD) and intermittent fault detection and isolation system (IFDIS) to detect intermittent faults on ground, including intermittent faults induced by joint crack, contact corrosion, loose plug/seat, loose connection and hairline cracks of circuit board line [22]. However, it still relies on laboratory conditions can be detected for intermittent faults induced by other causes/mechanisms, such as the tin/metal whisker.

\subsection{Diagnosis/Prediction Method}

In [23], the probability statistics method is used for the characteristics of intermittent faults to determine the probability value of the upper and lower bounds of the failed unit, and a method can be used to diagnose the permanent fault and intermittent fault of the scanning link. In order to meet the requirements of intermittent fault diagnosis, a new self-feedback observer is designed and applied to detect and diagnose sensor intermittent faults in [24]. In [25], the performance of environmental load and in-situ measurement is combined with life consumption monitoring and prediction algorithms of uncertainty correction to achieve the prediction of intermittent faults in digital systems. In [14, 15], based on the study of the dynamic characteristics of intermittent faults, in order to achieve the effect of intermittent fault diagnosis and prediction, the number of intermittent faults and the duration of the relay elements are kept statistics and the intensity of faults is measured by the temporary fault density and pseudo period in the time window. In [16], the methods of diagnosing and predicting intermittent faults are determined according to various filtering algorithms on the basis of state equation model.

\section{Analysis of Shortcomings of Intermittent Faults In Electronic Systems}

The intermittent fault of the electronic system although has achieved considerable development, it is still lack in many aspects, such as FMMEA, the selection of test analysis and detection method, general recognition of the intermittent fault and maintenance decision for the intermittent fault, specifically including the following 4 aspects. (1) The FMMEA system of intermittent faults is not perfect. The cause, mechanism, mode, and fault model described in section 2.1 above are part of the FMMEA (Failure Mode, Mechanism, and Effect Analysis). But there is no relevant theoretical research for intermittent faults in the other part-effect analysis, which is need to be researched vigorously in the future. (2) Study on test analysis and the selection of detection method for intermittent faults is inadequate. For the intermittent fault, the purpose of the current theory and practice is eager to detect intermittent faults, and relevant detection methods are studied a lot and have mature applications. Nevertheless, the study on test analysis and the selection of detection method for intermittent faults hasn't been carried out. (3) There exist deviations in general recognition of the intermittent fault. Although the further research on the fault cause, mechanism and mode, the general recognition of the intermittent fault hasn't developed and followed up with it. The early recognitions of the intermittent fault are remained in many views, and some of them even mislead the modeling methods and diagnostic methods for intermittent faults. (4) There is a lack of maintenance 
decision for intermittent faults. Confusing intermittent faults are intertwined with other factors that have led to a large number of NFF events, so the dangers of intermittent faults has been realized for a long time in the field of maintenance. The intermittent fault still is regarded as the major source of future NFF events after NFF being analyzed in detail, not only because the recognition, detection means and maintenance means of the intermittent fault are not advanced enough yet, but also because the maintenance decision method for intermittent faults effectively is inadequate.

\section{Conclusion}

In reality, the intermittent fault of the electronic system is often misdiagnosed as a permanent failure, resulting in unnecessary repeated machine halt, over-repair, and large wear parts and affecting the combat readiness of the equipment. In the paper, the concept and the research significance of intermittent faults are summarized, and the current research trends are analyzed. Generally speaking, compared with the research on permanent fault diagnosis, there are few research results of intermittent faults. The existing results are mostly in accordance with the traditional fault diagnosis ideas, only adding the priori information of intermittent faults to the traditional fault diagnosis method or simply regarding the intermittent fault as stochastic disturbances of the system to avoid the essential difficulties of intermittent fault diagnosis, such as strong randomness and short intermittent. At present, there isn't research results of the intermittent fault for the electronic system, which is systematic and has the universal significance. This paper focuses on the analysis of the intermittent fault for the electronic system from four aspects: the fault mode, the cause, the mechanism analysis, the modeling method, the detection method and the diagnosis/prediction method, so as to give some reference and inspiration to the research on the intermittent fault of electronic system.

\section{Acknowledgments}

This work was supported in part by the National Natural Science Foundation of China through grant 61502534, China Postdoctoral Science Foundation Funded Project through grant 2016M603052 and the Open Funds for the Joint Laboratory of China satellite measurement and control center through grant FOM2015OF015.

\section{References}

[1]. G. Deng, J. Qiu, Z. Li, a Survey on Intermittent Fault Diagnosis Technology, Ordnance Industry Automation. 40 (2015) 161-171.

[2]. Q. Haiyu, S. Ganesan, M. Pecht, No-Fault-Found and intermittent failures in electronic products, Microelectron. Reliab, 48 (2008) 663-674.

[3]. K. Rogers, C. Hillman, M. Pecht, Hollow Fibers Can Accelerate Conductive Filament Formation, ASM International Practical Failure Analysis, 1 (2011) 57-60.

[4]. C. Courbon, T. Mabrouki, J. Rech, D. Mazuyer, E. D'Eramo. On the existence of a thermal contact resistance at the tool-chip interface in dry cutting of AISI 1045: Formation mechanisms and influence on the cutting process, Appl. Therm. Eng, 50 (2013) 1311-1325.

[5]. Y. Gorash, H. F. Chen, Creep-fatigue life assessment of cruciform weldments using the linear matching method, Int. J. Pres. Ves. Pip, 104 (2013) 1-13.

[6]. S. W. Zou, X. G. Li, C. F. Dong, Electrochemical migration, whisker formation, and corrosion behavior of printed circuit board under wet H2S environment, Electrochimica Acta, 114 (2013) 363-371.

[7]. W.B. Ren, P. Wang, J. Song, Effects of current load on wear and fretting corrosion of gold-plated electrical contacts, Tribology International, 170 (2014) 75-82. 
[8]. A. Skwarek, M. Pluska, A. Czerwinsk, Influence of laminate type on tin whisker growth in tinrich lead-free solder alloys, Materials Science and Engineering B, 177 (2012) 1286-1291.

[9]. C. Liu, J. Wang, A. Zhang, Research on the fault diagnosis technology of intermittent connection failure belonging to FPGA solder-joints in BGA package, Optik, 125 (2014) 737-740.

[10]. E. George, M. Pecht, Tin whisker analysis of an automotive engine control unit, Microelectron. Reliab, 54 (2014) 214-219.

[11]. L.W. Zhou, S. Q. Zhou, M. G. Xu, Investigation of gate voltage oscillations in an IGBT module after partial bond wires lift-off, Microelectron. Reliab, 53(2013) 282-287.

[12]. W. C. Maia Filho, M. Brizoux, H. Fremont, Y. Danto, Improved Physical Understanding of Intermittent Failure in Continuous Monitoring Metho, in the14th IPFA, pp.141-146, 2007.

[13]. A. Bondavalli, S. Chiaradonna, F. D. Giandomenico, Threshold-based mechanisms to discriminate transient from intermittent fault, IEEE T. Comput, 49 (2000) 230-245.

[14]. A. Das, A. Kumar, B. Veeravalli, Communication and Migration Energy Aware Design Space Exploration for Multicore Systems with Intermittent Faults, in the 2013 Design, Automation \& Test in Europe Conference \& Exhibition, pp. 1631-1636, 2013.

[15]. A. Correcher, E. Garcia, F. Morant, Intermittent Failure Dynamics Characterization, IEEE T. Reliab, 61, (2012) 649-658.

[16]. X. D. Wu, Z. H. Song, Multi-step prediction of chaotic time-series with intermittent failures based on the generalized nonlinear filtering methods, Appl. Math. Comput, 219, (2013) 85848594.

[17]. R. Abreu, P. Zoeteweij, J. C. Arjan, A New Bayesian Approach to Multiple Intermittent Fault Diagnosis, in the Twenty-First International Joint Conference on Artificial Intelligence, pp. 653658, 2009.

[18]. R. C. Parkey, C. Hughes, M. Caulfield, P. Michael, A Method of Combining Intermittent Arc Fault Technologies, IEEE Autotestcon, (2012) 244-249.

[19]. Y. C. Chung, C. Furse, J. Pruitt. Application of phase detection frequency domain reflectometry for locating faults in an f-18 flight control harness, IEEE T. Electromagn. C, 47 (2005) 327-334.

[20]. C. Furse, Y.C. Chung, C. Lo, P. Pendayala. A critical comparison of reflect methods for location of wiring faults, Smar. Struct . Syst, 2 (2006) 25-46.

[21]. R. White, B. Richardson, Anecdotal Experiences on the Value of Limited Environmental Testing for the Analysis of "No Fault Found" Assemblies, IEEE Autotestcon, pp.292-296, 2011.

[22]. Y. Huang, T. C. Wu, M. R. Sudhakar. Statistical Diagnosis for Intermittent Scan Chain HoldTime Fault, in ITC International Test Conference, pp. 319-328, 2003.

[23]. T. Sedighi, P. D. Foote, P. Sydor, Feed-forward observer-based intermittent fault detection, Cirp Journal of Manufacturing Science \& Technology, 2016, available online.

[24]. G. F. Zhang, C. Kwan, R. Xu, An Enhanced Prognostic Model for Intermittent Failures in Digital Electronics, in IEEE Aerospace Conference, pp. 1-8, 2007. 